\title{
Prevalência de agravos de pele e fatores associados em trabalhadores de uma empresa agropecuária do sul do Brasil
}

\author{
Prevalence of skin disease and associated factors \\ in workers of an agricultural company in Southern Brazil \\ Rafael Haeffner', Rita Maria Heck², Vanda Maria da Rosa Jardim²
}

\begin{abstract}
RESUMO | Contexto: Apesar da proteção conferida à saúde pela pele e anexos, há fatores que podem atingir esse órgão, provocando nele importantes agravos. Entre tais fatores estão os ocupacionais. Objetivo: Descrever a prevalência e analisar os fatores associados com problemas de pele, em geral autorrelatados, causados pelo trabalho. Métodos: O presente estudo, de corte transversal, entrevistou trabalhadores de uma empresa agropecuária do sul do Brasil. O instrumento da pesquisa foi um questionário semiestruturado com questões abertas e fechadas, aplicado por meio de entrevistas. Resultados: Dos 326 trabalhadores da empresa, foram entrevistados 273 (83,8\%), e foi encontrada uma prevalência de agravos de pele de $15,2 \%$. Houve maior ocorrência de problemas de pele em homens (16,8\%), faixa etária dos 41 aos 50 anos (20,3\%), associados com exposições ocupacionais, especialmente a poeiras. Conclusão: Conclui-se que é importante o gerenciamento dos riscos ambientais, uma vez que podem estar associados ao surgimento de problemas de pele relacionados ao trabalho.

Palavras-chave I dermatose ocupacional; saúde do trabalhador; exposição ocupacional; epidemiologia; produção agrícola.

ABSTRACT I Context: Despite the health protection provided by the skin and appendages, there are factors that can affect this organ, causing major skin injuries. Among such factors are the occupational ones. Objective: To describe the prevalence and to analyze the factors associated with generally self-reported skin problems caused by work. Methods: This cross-sectional study interviewed workers from an agricultural company in Southern Brazil. The research instrument was a semi-structured questionnaire containing opened and closed questions. Results: Out of the 326 employees in the company, 273 (83.8\%) were interviewed. A prevalence of skin disorders of $15.2 \%$ was found. There was a greater occurrence of skin problems in men (16.8\%), who were aged between 41 and 50 years $(20.3 \%)$. These problems were associated with occupational exposures, especially to dust. Conclusion: It can be concluded that environmental risk management is important, as it may be associated with the occurrence of work-related skin problems.

Keywords I dermatitis, occupational; occupational health; occupational exposure; epidemiology; crop production.
\end{abstract}




\section{INTRODUÇÃO}

A pele é um órgão essencial ao organismo humano, sendo responsável por algumas nobres funções, como proteção física e imunológica, percepção, secreção e termo regulação ${ }^{1}$. O cuidado com a pele é vital para a saúde, inclusive no ambiente e processo de trabalho das pessoas.

A expansão das economias no mundo gerou mudanças e a alta da produtividade, o que eleva as cargas de exposições ocupacionais a que os trabalhadores estão submetidos no cotidiano diário de trabalho. Isso pode resultar no aumento e agravamento das doenças de origem ocupacional, inclusive as relacionadas à pele ${ }^{2}$.

Os agravos de pele variam desde leves sintomas (como dermatite de contato e lesões abrasivas causadas por objetos ou instrumentos de trabalho), até os variados tipos de câncer de pele $\mathrm{e}^{1,3}$.

As doenças de pele e anexos representam uma parcela considerável dos agravos ocupacionais em geral. Entretanto, há poucos dados estatísticos reais sobre o problema, pois em muitos casos tais agravos são autotratados pelo paciente, ou atendidos a nível ambulatorial sem gerar dados, sendo assim, os poucos números existentes podem apenas representar a ponta do iceberg ${ }^{1}$.

A prevalência dos problemas de pele, especialmente os relacionados ao trabalho, muda conforme aspectos citados anteriormente e a localidade ou país do contexto dos trabalhadores ${ }^{4}$. Pesquisas ocupacionais realizadas na Ásia demonstraram que a prevalência de sintomas de pele relacionados ao trabalho variou de 12 a $28 \%$,

As doenças de pele relacionadas ao trabalho podem variar conforme o grupo ocupacional do trabalhador, pois o tipo e o grau da exposição a fatores de risco difere entre ocupações ${ }^{2}$. Tais doenças estão diretamente associadas aos mais distintos fatores de risco do ambiente em que os trabalhadores estão expostos, como radiações, umidade relativa do ar, temperatura, microrganismos, produtos químicos, entre outros ${ }^{1,2,7,8}$.

As pesquisas que analisam os problemas de pele relacionados ao trabalho no Brasil são escassas, contudo a força de trabalho só aumenta no país, e hoje é de aproximadamente 96 milhões de trabalhadores, por isto justificam-se pesquisas deste cunho9. Com isso, o objetivo do presente estudo foi descrever a prevalência e analisar os fatores associados com problemas de pele relacionados ao trabalho.

\section{MÉTODOS}

O presente estudo, de abordagem quantitativa, observacional, com delineamento de corte transversal, fez parte de um projeto de pesquisa da Faculdade de Enfermagem da Universidade Federal de Pelotas. Foi realizado em uma empresa agropecuária de médio porte, localizada no sul do Brasil, e possui vínculo com o Ministério da Agricultura, Pecuária e Abastecimento.

Os participantes do estudo eram todos trabalhadores da referida empresa, com idade a partir de 18 anos. Para inclusão dos participantes foram seguidos os seguintes critérios: ser trabalhador efetivo da empresa e ter assinado o Termo de Consentimento Livre e Esclarecido (TCLE). Os trabalhadores eram das seguintes áreas: assistência administrativa, supervisão/gerenciamento, operacional, técnico científico, técnico assistencial, pesquisa e serviços gerais. Os setores foram divididos em: apoio, pesquisa, laboratório e campo (Quadro 1).

$\mathrm{O}$ instrumento semiestruturado foi multidimensional, composto por: questões sociodemográficas, estilo de vida, exposições, cargas ocupacionais e condições de trabalho. Foram incluídas também as questões do instrumento Percepção de Morbidade Geral e Percepção de Morbidade Relacionada com o Trabalho ${ }^{10}$. As entrevistas foram realizadas na empresa durante o turno de trabalho com prévia autorização da direção da empresa. Para a coleta de dados, foram capacitados acadêmicos de enfermagem. Os dados foram coletados no período compreendido entre os meses de junho e outubro de 2008.

Foi construído um banco de dados com as informações coletadas por meio dos questionários aplicados. Os dados foram inseridos por dupla digitação independente, utilizando-se o software EpiInfo versão 6.04. Após a verificação e o controle de erros e inconsistências, a análise dos dados foi realizada no software Stata versão 12.

As variáveis analisadas foram sexo, idade/faixa etária ( $\leq 44$ anos, $45-50$ anos, e $\geq 51$ anos), cor da pele (branco, negro, amarelo e pardo). Os grupos ocupacionais, setores de trabalho, tempo de trabalho na ocupação ( $\leq 72$ meses, 73-240 meses, e $\geq 241$ meses) também foram analisados.

As exposições ocupacionais foram norteadas pela seguinte questão (variáveis independentes): Em seu trabalho, o(a) $\operatorname{Sr}(\mathrm{a})$. enfrenta as seguintes condições ( $\mathrm{sim} /$ não): calor excessivo?; frio excessivo?; pó?; ar condicionado?; radiações?; 
trabalhar a "céu aberto"?; umidade excessiva?; ficar "molhado" ou "sujo"?; problemas de pele nos últimos 30 dias?

A questão referente ao agravo ocupacional investigado (variável dependente) foi a seguinte: $\mathrm{O}(\mathrm{a}) \mathrm{Sr}(\mathrm{a})$. acha que o seu trabalho costuma causar lhe problemas de pele ( $\mathrm{sim} /$ não)?

Para a análise dos dados foram utilizadas frequência absoluta e relativa para as variáveis socioeconômicas, demográficas e de organização de trabalho, e ainda calculado o intervalo de confiança de 95\% (IC95\%) para o agravo ocupacional “agravos de pele relacionados ao trabalho". Foram calculadas também a média, mediana, valor máximo e mínimo, variância e desvio padrão para as variáveis contínuas.

Foi realizado o cálculo da Razão de Prevalência (RP) para obtenção da medida de efeito para associação com IC95\% entre a variável "agravo de pele relacionado ao trabalho", e as variáveis de exposições ocupacionais autorreferidas (calor excessivo, frio excessivo, ar condicionado, pó, radiações, trabalhar a "céu aberto", umidade, ficar molhado ou sujo ao realizar as tarefas de trabalho) ${ }^{11}$. Foram verificadas as quatro variáveis de exposição com maior significância e com $p<0,20$ na análise univariada, para inclusão no modelo múltiplo.

Após a análise bruta da RP, foi realizado o modelo múltiplo com Regressão de Poisson ${ }^{11}$ pelo modelo step wise forward entre a variável desfecho, e as variáveis de exposição ocupacional, considerando significativo $\mathrm{p}<0,05$ e descritos os IC $95 \%$.
Os testes do $\chi^{2}$ de Pearson ou exato de Fisher foram utilizados para comparar a diferença de dermatose ocupacional quanto ao sexo, faixa etária, cor da pele, problemas de pele nos últimos 30 dias, grupos ocupacionais, setores de trabalho dos trabalhadores, tempo de trabalho na ocupação, tendo sido considerado significativo $\mathrm{p}<0,05$.

O projeto de pesquisa foi enviado ao Comitê de Ética em Pesquisa da Faculdade de Odontologia da UFPEL, em conformidade com a Resolução 196/96 do Conselho Nacional de Saúde (CNS), e obteve o parecer favorável sob o n. ${ }^{\circ} 011 / 2008$ no dia 18 de abril de 2008.

\section{RESULTADOS}

No período das entrevistas, a empresa contava com 326 trabalhadores, dentre os quais 304 exerciam as atividades nas dependências da empresa. Os demais estavam afastados para aperfeiçoamento profissional e viagens técnicas, licença para tratamento de doença e férias. Entre as perdas e recusas participaram desta pesquisa $273(83,8 \%)$ trabalhadores.

Quanto às características sociodemográficas da referida empresa, foi identificada predominância do sexo masculino $(79,1 \%)$, e da faixa etária dos 41 aos 50 anos $(45,8 \%)$, sendo a média de idade dos trabalhadores de 47,6 anos, a mediana

Quadro 1. A descrição dos grupos ocupacionais e setores de trabalho da referida empresa, sul do Brasil, 2008.

\begin{tabular}{|c|c|}
\hline \multicolumn{2}{|r|}{ Grupos Ocupacionais } \\
\hline Assistência administrativa & Secretariado, auxiliar e agente administrativo, e assessores de chefias. \\
\hline Supervisão & Supervisores, gerentes e gestores da empresa. \\
\hline Operacional & $\begin{array}{l}\text { Operadores de máquinas e veículos, operadores em geral, motoristas, porteiros, } \\
\text { vigilantes, telefonistas, campeiro e "trabalhadores braçais". }\end{array}$ \\
\hline Pesquisa & Pesquisadores das diversas áreas da empresa. \\
\hline Técnico científico & Trabalhadores com formação acadêmica. \\
\hline Técnico assistencial & Trabalhadores de nível médio com curso técnico em alguma área de atuação. \\
\hline Serviços gerais & Trabalhadores responsáveis pela limpeza e higienização da empresa. \\
\hline \multicolumn{2}{|r|}{ Setores de Trabalho } \\
\hline Apoio & $\begin{array}{c}\text { Setores referentes às áreas de administração, gestão, economia, financeira, comunicação, } \\
\text { marketing, cultura, compras e recursos humanos da empresa. }\end{array}$ \\
\hline Pesquisa & $\begin{array}{c}\text { Referente às diferentes áreas de formação envolvidas com a pesquisa agropecuária - como a pesquisa } \\
\text { animal e vegetal, melhoramento genético, agroecologia, climatologia e agrometeorologia. }\end{array}$ \\
\hline Laboratórios & Referente aos mais distintos laboratórios de análises das pesquisas da empresa. \\
\hline Campo & $\begin{array}{l}\text { Engloba todo o trabalho realizado no campo, referente ao tratamento de animais em pesquisa; } \\
\text { plantio, cultivo, colheita dos espécimes vegetais pesquisadas. }\end{array}$ \\
\hline
\end{tabular}


de 49 anos, o desvio padrão (DP) de 8,2 anos, com idade mínima de 19 e máxima de 69 anos, e a variância de 68,7 anos (Tabela 1). Em relação ao tempo de trabalho na função, a média foi de 167,3 meses, mediana de 132,0, variância de $16.183,2$, e DP de 127,2 meses, com valor mínimo de 1 e máximo 624 meses.

Agravos de pele autorreferidos causados pelo trabalho na empresa pesquisada tiveram prevalência de 15,2\% (IC95\% $11,1-20,0)$, atingiram mais o sexo masculino (16,8\%), na faixa etária dos 45 aos 50 anos de idade $(\mathrm{p}=0,038)$. Quanto à cor da pele, pardos $(25,0 \%)$ e brancos $(15,6 \%)$ foram os mais acometidos. Entretanto não houve diferença significativa entre os grupos sociodemográficos. A prevalência de agravos de pele - relacionados significativamente ao trabalho $(\mathrm{p}<0,001)$ - foi de $9,6 \%$ nos 30 dias anteriores (recorrência) à entrevista (Tabela 1 ).

Em relação aos grupos ocupacionais, o grupo ocupacional da pesquisa foi o menos acometido por problemas de pele (4,5\%). Enquanto os grupos ocupacionais mais atingidos foram os técnico científico, técnico assistencial e serviços gerais. Entre esses a prevalência pouco variou
$(23,1$ a $24,1 \%)$. Entre os setores houve diferença significativa $(\mathrm{p}=0,02)$, sendo o setor de campo o mais acometido $(25,0 \%)$ (Tabela 2$)$.

Quanto ao tempo de trabalho na empresa, somente os trabalhadores com menos de um ano de trabalho não tiveram agravos de pele, em outras faixas de tempo a diferença variou de 10,4 a 18,5\%. A faixa de tempo com menor prevalência de agravos de pele foi de até 6 anos trabalhando na mesma função $(10,4 \%)$ e com maior prevalência foi de 6 a 20 anos (18,5\%). Houve associação significativa para a variável setor de trabalho, e uma tendência de associação para grupo ocupacional (Tabela 2).

As variáveis de exposição mais associadas com agravos de pele foram: o pó com RP de 5,10 (IC95\% 2,15-12,13); ficar molhado ou sujo em função das atividades laborais, com RP de 2,90 (IC95\% 1,56-5,40); calor excessivo, com RP de 2,68 (IC95\% 1,37-5,25); e frio excessivo, com RP de 2,34 (IC95\% 1,25-4,39). Já no modelo ajustado final a única variável com efeito independente para o desfecho foi exposição ao pó, sendo ajustada em mais de 10,0\% pela variável "ficar molhado ou sujo" durante as tarefas laborais (Tabela 3).

Tabela 1. Prevalência de agravos de pele dos trabalhadores da empresa agropecuária, conforme as variáveis de sexo, faixa etária, cor da pele e problemas de pele nos 30 dias anteriores à entrevista, sul do Brasil, 2008 ( $n=273$ ).

\begin{tabular}{|c|c|c|c|c|}
\hline \multirow{3}{*}{ Variável } & \multirow{3}{*}{ n (\%) } & \multicolumn{2}{|c|}{ Problemas de pele } & \multirow{3}{*}{ Valor $\mathrm{p}$} \\
\hline & & Sim & Não & \\
\hline & & n (\%) & n (\%) & \\
\hline \multicolumn{4}{|l|}{ Sexo } & \multirow{3}{*}{$0,127^{*}$} \\
\hline Masculino & $216(79,1)$ & $37(17,2)$ & $178(82,8)$ & \\
\hline Feminino & $57(20,9)$ & $5(8,9)$ & $51(91,1)$ & \\
\hline \multicolumn{4}{|c|}{ Faixa etária (anos) } & \multirow{4}{*}{$0,038^{*}$} \\
\hline$\leq 44$ & $83(30,4)$ & $12(14,6)$ & $70(85,4)$ & \\
\hline $45-50$ & $90(33,0)$ & $20(22,5)$ & $69(77,5)$ & \\
\hline$\geq 51$ & $100(36,6)$ & $9(9,1)$ & $90(90,9)$ & \\
\hline \multicolumn{4}{|l|}{ Cor de pele } & \multirow{5}{*}{$0,220^{* *}$} \\
\hline Branco & $241(88,3)$ & $37(15,5)$ & $201(84,5)$ & \\
\hline Negro & $14(5,1)$ & $\mathrm{O}(0,0)$ & $14(100,0)$ & \\
\hline Pardo & $16(5,9)$ & $4(25,0)$ & $12(75,0)$ & \\
\hline Amarelo & $2(0,7)$ & $\mathrm{O}(0,0)$ & $2(100,0)$ & \\
\hline \multicolumn{4}{|c|}{ Problemas pele nos 30 dias enteriores à entrevista } & \multirow{3}{*}{$<0,001^{*}$} \\
\hline $\operatorname{Sim}$ & $26(9,6)$ & $13(50,0)$ & $13(50,0)$ & \\
\hline Não & $245(90,4)$ & $28(11,5)$ & $216(88,5)$ & \\
\hline
\end{tabular}

*Teste do $\chi^{2}$ de Pearson; **Teste exato de Fisher. 


\section{DIsCusSÃo}

A prevalência de agravos de pele encontrada no estudo foi de $15,2 \%$ (IC95\% 11,1-20,0), e se associou principalmente à recorrência de tais agravos nos 30 dias anteriores à entrevista, setor de trabalho e exposição a fatores ambientais do trabalho (como o pó).

Em uma pesquisa com pacientes no Canadá, a dermatose ocupacional prevaleceu em $82,0 \%$ do total de doenças de pele diagnosticadas ${ }^{12}$. Na Polônia a prevalência de doenças de pele relacionadas ao trabalho variou de 21,0 a $67,0 \%$ conforme a ocupação exercida. Na Escócia, 31,0\% dos trabalhadores relataram pelo menos um sintoma de doença de pele ${ }^{13,14}$. Em estudo realizado com trabalhadores em Taiwan, a prevalência de doenças de pele variou de 27,0 a $28,2 \%$, resultado compatível com o achado em outro estudo, feito na Itália, o qual indicou que doenças de pele acometeram $25,0 \%$ dos trabalhadores ${ }^{15}$. E, na Indonésia, doenças de pele relacionadas ao trabalho atingiram $12,0 \%$ dos trabalhadores ${ }^{6}$, dado semelhante aos resultados encontrados no presente estudo.

Tabela 3. Análise bruta e ajustada para os estratos "sexo" e "idade", da associação de agravos de pele conforme as variáveis das exposições ocupacionais, sul do Brasil, 2008.

\begin{tabular}{l|c|c}
\multirow{2}{*}{ Variáveis } & Análise bruta & Análise ajustada \\
\cline { 2 - 3 } & RP (IC95\%) & RP* (IC95\%) \\
\hline Pó & $5,10(2,15-12,13)$ & $3,91(1,54-9,95)$ \\
\hline $\begin{array}{l}\text { Ficar molhado } \\
\text { Ou sujo }\end{array}$ & $2,90(1,56-5,40)$ & $1,70(0,87-3,32)$ \\
\hline Calor excessivo & $2,68(1,37-5,25)$ & ns \\
\hline Frio excessivo & $2,34(1,25-4,39)$ & nS \\
\hline
\end{tabular}

RP: razão de prevalência; ns: variáveis que perderam significância no modelo final; IC95\%: intervalo de confiança de 95\%; *Razão de prevalência ajustada por regressão de Poisson.

Tabela 2. Prevalência de agravos de pele dos trabalhadores da empresa agropecuária, conforme as variáveis de grupo ocupacional, setor de trabalho, tempo de trabalho na empresa, tempo de trabalho na função, sul do Brasil, 2008 (n=273).

\begin{tabular}{|c|c|c|c|c|}
\hline \multirow{3}{*}{ Variável } & \multirow{3}{*}{ n (\%) } & \multicolumn{2}{|c|}{ Problemas de pele } & \multirow{3}{*}{ Valor $\mathrm{p}$} \\
\hline & & Sim & Não & \\
\hline & & $\mathrm{n}(\%)$ & $\mathrm{n}(\%)$ & \\
\hline \multicolumn{4}{|l|}{ Grupo ocupacional } & \multirow{8}{*}{$0,061^{*}$} \\
\hline Assistência administrativa & $29(10,7)$ & $4(14,3)$ & $24(85,7)$ & \\
\hline Supervisão/gerenciamento & $18(6,6)$ & $3(16,7)$ & $15(83,3)$ & \\
\hline Operacional & $57(20,9)$ & $7(12,7)$ & $48(87,3)$ & \\
\hline Pesquisa & $67(24,6)$ & $3(4,5)$ & $64(95,5)$ & \\
\hline Técnico científico & $26(9,6)$ & $6(23,1)$ & $20(76,9)$ & \\
\hline Técnico assistencial & $62(22,8)$ & $15(24,2)$ & $47(75,8)$ & \\
\hline Serviços gerais & $14(4,8)$ & $3(23,1)$ & $10(76,9)$ & \\
\hline \multicolumn{4}{|l|}{ Setor de Trabalho } & \multirow{6}{*}{$0,020^{*}$} \\
\hline Apoio & $115(42,9)$ & $16(14,1)$ & $98(85,9)$ & \\
\hline Pesquisa & $65(24,3)$ & $4(6,1)$ & $61(93,9)$ & \\
\hline Laboratório & $38(14,2)$ & $9(23,7)$ & $29(76,3)$ & \\
\hline Campo & $50(18,7)$ & $12(25,0)$ & $36(75,0)$ & \\
\hline Registros perdidos & 5 & & & \\
\hline \multicolumn{4}{|c|}{ Tempo de trabalho na função (meses) } & \multirow{4}{*}{$0,260^{*}$} \\
\hline$\leq 72$ & $97(35,5)$ & $10(10,4)$ & $86(89,6)$ & \\
\hline $73-240$ & $93(34,1)$ & $17(18,5)$ & $75(81,5)$ & \\
\hline$\geq 241$ & $83(30,4)$ & $14(17,1)$ & $68(82,9)$ & \\
\hline
\end{tabular}

*Teste do $\chi^{2}$ de Pearson. 
Em pesquisa realizada em uma clínica de dermatologia no Brasil, 10,0\% dos pacientes pesquisados apresentaram dermatite ocupacional de contato, o que representa $80,0 \%$ das doenças de pele relacionadas ao trabalho ${ }^{7}$. Em trabalhadores rurais de Campinas (SP), aproximadamente 10,0\% tiveram alterações dermatológicas possivelmente devido a exposição combinada de múltiplos agrotóxicos ${ }^{16}$. Em outra pesquisa realizada com trabalhadores no estado de São Paulo, a taxa de problemas dermatológicos foi de $27,0 \%{ }^{17}$.

A prevalência das doenças de pele relacionadas ao trabalho quanto ao sexo pode variar conforme o estudo. Em pesquisas ocupacionais que avaliaram sintomas de pele causados pelo trabalho, foi encontrada maior prevalência de tais sintomas em mulheres, e pode se associar significativamente com o sexo feminino ${ }^{3,5}$. Já em outras pesquisas os agravos de pele foram prevalentes entre os homens, e o sexo masculino foi considerado como fator de risco para dermatite ocupacional em trabalhadores da Califórnia ${ }^{4,7,12}$. A discordância de resultados entre os estudos citados, possivelmente ocorreu devido às diferenças entre as amostras e os ambientes em que os participantes estavam ${ }^{7}$.

A idade pode ser fator de associação de risco com problemas de pele, sendo risco em trabalhadores acima dos 30 anos de idade em ocupações não relacionadas à higienização, entretanto em trabalhadores de serviços de higienização a associação apresenta-se para faixa etária até 30 anos $^{5}$. Em pesquisa ocupacional realizada no Canadá, sintomas de pele foram encontrados com maior frequência em faixas etárias mais elevadas ${ }^{12}$. Já em uma revisão sistematizada, a faixa etária de maior risco para agravos ocupacionais de pele é dos 18 aos 24 anos $^{4}$.

Quanto à cor da pele uma pesquisa sobre dermatite ocupacional, demonstrou maior ocorrência do agravo em brancos $(46,0 \%)$, seguido de pardos $(39,0 \%)^{7}$. Em outro estudo com trabalhadores de engenharia de operação, a cor de pele branca foi associada a "queimaduras na pele causadas por exposição solar"3.

A ocupação e o setor de trabalho podem ser determinantes no surgimento dos agravos de pele ${ }^{18-20}$. Os trabalhadores de serviços gerais e higienização podem ter alta incidência de doenças de pele, além de ter risco de moderado a alto para tais agravos. Isso pode ocorrer devido aos tipos de exposições a que estão submetidos, e respectivas doses respostas ${ }^{4,7,13,14}$. Outros estudos ${ }^{4,7,8}$ mostraram que trabalhadores pertencentes a ocupações assistenciais e operacionais da indústria, construção civil e agricultura apresentam alta ocorrência e associação com doenças de pele, resultado semelhantes ao desse estudo.

O tempo de trabalho no setor e na ocupação é outro fator determinante para as doenças da pele, apesar da diferença não significativa dos resultados nesse estudo. Uma pesquisa realizada em Taiwan encontrou para o maior tempo de trabalho na ocupação o risco aumentado para agravos de pele, pois se reflete em maior tempo de exposição aos agentes agressores da pele ${ }^{5}$.

O ambiente de trabalho pode ter alguns fatores de riscos que causam doenças de pele, sendo eles os agentes biológicos, químicos e físicos. Entretanto os agentes químicos e biológicos costumam ser os maiores causadores de doenças de pele relacionadas ao trabalho $0^{6,8,12}$. A exposição ocupacional a poeiras e agentes químicos em geral (como óleos, solventes, produtos de higienização, tintas, resinas, metais, derivados de benzeno e do petróleo) pode causar diversos agravos à pele dos trabalhadores $^{6,8,12,15,21-23}$. Alguns produtos químicos utilizados em curtumes são perigosos à saúde humana, podendo causar alteração estrutural de pele e anexos dos trabalhadores ${ }^{6}$. Mas, a exposição prolongada do trabalhador a agentes físicos também pode associar-se a problemas de pele $e^{2,3}$. A exposição da pele à radiação solar, por exemplo, pode causar lesões por queimaduras ${ }^{2,3}$. E, conforme pesquisas, há outros agentes físicos que podem ser precursores de doenças de pele, como umidade e temperaturas extremas de calor ou frio excessivos ${ }^{5,20,21}$.

No Brasil existem leis e normas regulamentadoras do trabalho que fazem parte das políticas públicas em Saúde do Trabalhador, entre elas está a Norma Regulamentadora 6 (NR-6), que dispõe sobre a utilização de equipamentos de proteção individual (EPI), um conjunto de dispositivos contra um ou mais riscos ocupacionais, e podem prevenir ou reduzir a incidência de agravos relacionados ao trabalho, como os problemas de pele ${ }^{24}$.

Entre as limitações do presente estudo, podemos citar as perdas e o tamanho da amostra, o que pode ter influenciado a não associação de algumas variáveis de exposição ao desfecho, o que ocorreu em outras pesquisas com maior poder inferencial. Outra limitação é 
o delineamento do estudo, por ser transversal, possibilita apenas associações. E algumas vezes por ser transversal pode ocorrer o efeito da causalidade reversa, tendo em vista a questão da falta de temporalidade. Porém, é difícil que tenha ocorrido o efeito do "trabalhador sadio" uma vez que as perdas ocorreram quase em sua totalidade devido à licença para capacitação profissional, férias ou viagens de trabalho.

No período da pesquisa, a empresa possuía Serviço Especializado em Engenharia de Segurança e em Medina do Trabalho (SESMT) e Comissão Interna de Prevenção a Acidentes (CIPA). O SESMT era composto por dois técnicos em segurança do trabalho, um médico do trabalho e um engenheiro do trabalho. Além disto, atendiam a NR-6, disponibilizando EPI e monitorando as práticas laborais dos trabalhadores na medida do possível, ainda tinha PCMSO e PPRA. A empresa contava com 3 subestações de trabalho além de ocupar uma área territorial extensa em função do ramo de atividade econômica atuante, fator que possivelmente dificultasse o monitoramento simultâneo e constante de todos os trabalhadores.

\section{CONCLUSÃO}

Esta pesquisa encontrou uma prevalência relativamente baixa de agravos de pele relacionados ao trabalho, se comparada a resultados de outros estudos da literatura. Sendo que tais agravos tiveram maior associação com fatores como a faixa etária dos 45 aos 50 anos, recorrência de agravos de pele, setor de trabalho com maior proporção de agravos de pele e a exposição à poeira.

Os principais resultados do presente estudo viabilizam a elaboração de ações e estratégias iniciais para a prevenção de agravos de pele relacionados ao trabalho. Há uma necessidade de esforços integrados como a equipe SESMT, CIPA e trabalhadores da empresa, em consonância com as normas regulamentadoras dos ambientes de trabalho e políticas públicas em saúde do trabalhador.

Concluiu-se que é necessário que se façam novos estudos em torno da temática, com método capaz de estabelecer causa e efeito entre fatores sociodemográficos e do ambiente de trabalho, além da utilização de tecnologias capazes de identificar as cargas ocupacionais e mensurar as doses respostas, com objetivo da aproximação causal.

\section{REFERÊNCIAS}

1. Brasil. Ministério da Saúde. Secretaria de Atenção a Saúde. Departamento de Ações Programáticas Estratégicas. Saúde do Trabalhador. Dermatoses Ocupacionais. Brasília (DF), 2009; 92 p. [acesso em 2014 mar]. Disponível em: http://bvsms.saude.gov. br/bvs/publicacoes/protocolo_dermatoses.pdf

2. Keegel T, Moyle M, DharmageS, Frowen K, Nixon R. The epidemiology of occupational contact dermatitis (1990-2007): a systematic review. Int J Dermatol. 2009;48(6):571-8.

3. Duffy SA, Choi SH, Hollern R, Ronis DL. Factors associated with risky sun exposure behaviors among operating engineers. Am J Ind Med. 2012;55(9):786-92.

4. Irby CE, Yentzer BA, Vallejos QM, Arcury TA, Quandt SA, Feldman SR. The prevalence and possible causes of contact dermatitis in farmworkers. Int J Dermatol. 2009;48(11):1166-70.

5. Su SB, Wang BJ, Tai Chien, Chang HF, Guo HR. Higher prevalence of dry symptoms in skin, eyes, nose and throat among workers in clean rooms with moderate humidity. J Occup Health. 2009;51(4):364-9.

6. Febriana SA, Jungbauer F, Soebono H, Coenraads PJ. Inventory of the chemicals and the exposure of the workers' skin to these at two leather factories in Indonesia. Int Arch Occup Environ Health. 2012;85:517-26
7. Duarte I, Rotter A, Lazzarini R. Frequência da dermatite de contato ocupacional em ambulatório de alergia dermatológica. An Bras Dermatol. 2010;85(4):455-9.

8. Ahn YS, Kim MG. Occupational skin diseases in Korea. Korean Med Sci. 2010;25:S46-52.

9. Brasil. Instituto Brasileiro de Geografia e Estatística (IBGE). Banco de dados agregados: Pesquisa nacional por amostra de domicílios (PNAD): pesquisa básica, 2014. [acesso em 2014 out 10]. Disponível em: http://www.sidra.ibge.gov.br/bda/tabela/protabl. asp?c $=1869 \& z=$ pnad\&o=3\&i $=\mathrm{P}$

10. Fassa AG. O Trabalho como determinante de morbidade comum em uma indústria de celulose e papel [dissertação]. Pelotas (RS): Universidade Federal de Pelotas, Programa de Pós-Graduação em Epidemiologia; 1995.

11. Francisco PMSB, Donalisio MR, Barros MBA, Cesar CLG, Carandina L, Goldbaum M. Medidas de associação em estudo transversal com delineamento complexo: razão de chances e razão de prevalência. Rev Bras Epidemiol. 2008;11(3):347-55.

12. Arrandale VH, Kudla I, Kraut AG, Scott JA, Tarlo SM, Redlich CA, et al. Skin and respiratory symptoms among workers with suspected work-related disease. Occup Med. 2012;62:420-6. 
13. Mirabelli MC, Vizcaya D, Margarit AM, Antó JM, Arjona L, Barreiro E, et al. Occupational risk factors for hand dermatitis among professional cleaners in Spain. Contact Dermatitis. 2012;66(4):188-96.

14. Steiner MFC, Dick FD, Scaife AR, Semple S, Paudyal P, Ayres JG. High prevalence of skin symptoms among bakery workers. Occup Med. 2011;61:280-2.

15. Magnavita N, Elovainio M, Heponiemi T, Magnavita AM, Bergamaschi A. Are skin disorders related to work strain in hospital workers? A cross-sectional study. BMC Public Health. 2011;11:600.

16. Figueiredo GM, Trape AZ, Alonzo HA. Exposição a múltiplos agrotóxicos e prováveis efeitos a longo prazo à saúde: estudo transversal em amostra de 370 trabalhadores rurais de Campinas (SP). Rev Bras Med Trab. 2011;9(1):1-9.

17. Kaddourah SMH, Costa FAA, Fábrega JF, Rabello LVA. Relação entre a ocorrência de dermatite de contato irritativa e o uso dos equipamentos de proteção individual. Rev Bras Med Trab. 2015;13(2):120-5.

18. Fantuzzi G, Righi E, Predieri G, Giacobazzi P, Mastroianni K, Aggazzotti G. Prevalence of ocular, respiratory and cutaneous symptoms in indoor swimming pool workers and exposure to disinfection by-products (DBPs). Int J. Environ Res Public Health. 2010;7:1379-91.
19. Arrandale V, Meijster T, Pronk A, Doekes G, Redlich CA, Holness DL, et al. Skin symptoms in bakery and auto body shop workers: associations with exposure and respiratory symptoms. Int Arch Occup Environ Health. 2013;86:167-75.

20. Kurpiewska J, Liwkowicz J, Benczek K. A survey of work-related skin diseases in different occupations in Poland. Int J Occup Saf Ergon. 2011;17(2):207-14.

21. HolnessDL,Kudlal.Workerswithoccupational contactdermatitis:workplace characteristics and prevention practices. Occup Med. 2012;62:455-7.

22. Tresukosol P, Swasdivanich C. Hand contact dermatitis in hairdressers: clinical and causative allergens, experience in Bangkok. Asian Pac J Allergy Immunol. 2012;30:306-12.

23. Sripaiboonkij P, Sripaiboonkij N, Phanprasit W, Jaakkola MS. Respiratory and skin health among glass microfiber production workers: a cross-sectional study. Environ Health. 2009;8:36.

24. Brasil. Norma Regulamentadora 6 (Atualização). Equipamento de Proteção Individual. Brasília (DF): Ministério do Trabalho e Emprego; 2010. [acesso em 2014 jun 30]. Disponível em: http://portal.mte.gov. br/data/files/FF8080812DC56F8F012DCDAD35721F50/NR-06\%20 (atualizada)\%202010.pdf

Endereço para correspondência: Rafael Haeffner - Rua Padre João Wislinski, 119, apto 22, bloco B, Santa Cândida - CEP: 82630-494 - Curitiba (PR), Brasil -

E-mail: rafaelhaeffner@gmail.com 